\title{
Short- and Long-Term Statistical Properties of Heartbeat Time-Series in Healthy and Pathological Subjects
}

\author{
Paolo Allegrini ${ }^{1}$, Rita Balocchi ${ }^{2}$, Santi Chillemi ${ }^{3}$, Paolo Grigolini ${ }^{3,4,5}$, Luigi \\ Palatella $^{4}$, and Giacomo Raffaelli ${ }^{6}$ \\ 1 Istituto di Linguistica Computazionale del Consiglio Nazionale delle Ricerche, Area \\ della Ricerca di Pisa-S. Cataldo, Via Moruzzi 1, 56124, Pisa, Italy. \\ (paolo.allegrini@ilc.cnr.it), \\ 2 Istituto di Fisiologia Clinica del Consiglio Nazionale delle Ricerche, Area della \\ Ricerca di Pisa-S. Cataldo, Via Moruzzi 1, 56124, Pisa, Italy. \\ ${ }^{3}$ Istituto di Biofisica del Consiglio Nazionale delle Ricerche, Area della Ricerca di \\ Pisa-S. Cataldo, Via Moruzzi 1, 56124, Pisa, Italy \\ 4 Dipartimento di Fisica dell'Università di Pisa and INFM, via Buonarroti 2, 56127 \\ Pisa, Italy. \\ ${ }^{5}$ Center for Nonlinear Science, University of North Texas, P.O. Box 311427, Denton, \\ Texas, 76203-1427 USA. \\ 6 International School for Advanced Studies and INFM Unit, via Beirut 2-4, 34014
} Trieste, Italy.

\begin{abstract}
We analize heartbeat time-series corresponding to several groups of individuals (healthy, heart transplanted, with congestive heart failure (CHF), after myocardial infarction (MI), hypertensive), looking for short- and long-time statistical behaviors. In particular we study the persistency patterns of interbeat times and interbeat-time variations. Long-range correlations are revealed using an information-based technique which makes a wise use of the available statistics. The presence of strong long-range time correlations seems to be a general feature for all subjects, with the exception of some CHF individuals. We also show that short time-properties detected in healthy subjects, and seen also in hypertensive and MI patients, and completely absent in the trasplanted, are characterized by a general behavior when we apply a proper coarsegraining procedure for time series analysis.
\end{abstract}

\section{Introduction}

In the last few years, time series analysis techniques have been applied to heartbeat sequences to detect long-range memory [1,2,5]. With these methods one can in principle distinguish between different statistical properties that may correspond to different pathophysiological condition. Another important goal of this analysis is to unravel the hidden control mechanisms responsible for the heartbeat dynamics. Indeed heart rate variability is influenced by the "competition" between sympathetic and parasympathetic nervous system activity as well as by 
non-autonomic factors. Consequently, an analysis regarding different time scales can in principle disentangle the different contributions to the heart rate variability. In this work we use a recently developed technique for measuring long-range correlations called Diffusion Entropy (DE) [4.5.6. We also focus on short-range time behavior and, using a coarse-graining procedure, we identify an objective resolution time for the measure of the interbeat distance. We use this results to compute the relative importance of the short-time dynamics with respect to the long-term one.

\section{Materials and methods}

\subsection{The diffusion entropy technique}

In order to process the data, the first step consists of a coarse-graining procedure on the RR time series. This strategy, shared also by other groups (see, for instance, Ref. [2]) makes a statistical treatment of the data possible.

We define a coarse-graining parameter $s$ and we obtain a new series, namely

$$
T_{i}(s) \equiv\left[T_{i} / s\right],
$$

where $T_{i}$ is the time distance between the $i^{t h}$ and the $i-1^{t h}$ beat, $T_{i}(s)$ is the coarse-grained time series and [.] denotes the integer part. Equation (11) means that we preliminary divide the interbeat distance scale in several "boxes" of size $s$ and we assign to all the interbeat distances lying in the same "box" the same value in the sequence $T_{i}(s)$. Next, we convert this series into a dichotomous sequence, setting $\xi_{i}=1$ when $T_{i}(s) \neq T_{i-1}(s)$, and $\xi_{i}=0$ if $T_{i}(s)=T_{i-1}(s)$. Finally, we generate several trajectories (labelled with the index $l$ ) for the variable $x_{l}$ at "time" $t$, namely,

$$
x_{l}(t)=\sum_{i=l}^{l+t} \xi_{i} .
$$

Note that, for simplicity, we have omitted indicating the dependence on $s$. As shown in Ref. [4], if the sequence $\xi_{i}$ is ergodic, then the probability distribution of the variable $x$ as a function of $t$ is expected to fit the scaling property

$$
p(x, t)=\frac{1}{t^{\delta}} F\left(\frac{x}{t^{\delta}}\right),
$$

with the "degree of anomaly" being measured by the distance of the scaling parameter $\delta$ from the standard value 0.5 corresponding to Brownian motion.

It is straightforward to prove that the Shannon entropy

$$
S(t)=-\int_{-\infty}^{\infty} p(x, t) \ln (p(x, t)) \mathrm{d} x
$$

of a process fitting the scaling condition of Eq. (3) yields

$$
S(t)=A+\delta \ln (t)
$$


where $A$ is a constant, whose explicit form is not relevant for the ensuing discussion. This result is immediately obtained by plugging Eq. (3) into Eq. (4). The above method of evaluating the scaling parameter $\delta$ is called Diffusion Entropy (DE), and, as it will become clear later, this technique is more efficient than the calculation of the second moment of the probability distribution. Note that when the distribution density under study departs from the ordinary Gaussian case and the function $F(y)$ has slow tails with an inverse power law nature [I] the second moment is a divergent quantity. This diverging quantity is made finite by the unavoidable statistical limitation. In this case, the second moment analysis would be determined by the statistical inaccuracy, thereby leading to misleading conclusions, while the method based on Eq. (5) yields correct results [ [6.5].

\subsection{Short-time analysis}

Let us define the sequence $\left\{\tau_{j}\right\}$ as the distance between 1's in the series $\left\{\xi_{i}\right\}$. The authors of Ref. [1] studied the case where the probability distribution for $\tau$ decays as an inverse-power law with exponent $\mu>2$. If $\tau_{j}$ were uncorrelated, then a simple expression would exists, linking $\mu$ and $\delta$, namely

$$
\delta=\frac{1}{\mu-1}(\delta=0.5 \text { if } \mu>3) .
$$

In our analysis we observe a strong discrepancy between the values of $\delta \approx 0.8$ and of $\mu \approx 5$, thus suggesting that the sequence of $\tau_{j}$ has a long time memory [6]. This is why we perform the normalized auto-correlation function of the sequence $\tau_{j}$ defined as

$$
\Phi_{\tau}(j)=\frac{\left\langle\left(\tau_{k}-\langle\tau\rangle\right)\left(\tau_{k+j}-\langle\tau\rangle\right)\right\rangle}{\left\langle\tau_{k}^{2}\right\rangle-\langle\tau\rangle^{2}}
$$

where $\langle\cdot\rangle$ is the arithmetic average, e.g.

$$
\langle\tau\rangle \equiv \frac{1}{N} \sum_{k=0}^{N} \tau_{k},
$$

and $N$ is the number of terms in the sum.

At this point we realize that for most data the correlation function $\Phi_{\tau}(j)$ actually decays as an inverse power law after an abrupt fall occurring at the transition from $j=0$ to $j=1$. The value at $j=1$, defined as $\epsilon^{2}$, exhibits a peculiar behavior as a function of the coarse-graining parameter $s$. We will show that this behavior is typical of all the groups, with the exception of transplanted patients and some CHF individuals.

\subsection{RR time series}

The RR series analyzed are taken from the NOnLinear TIme Series AnaLysIS (NOLTISALIS) archive. This data set is the result of the collaboration of several 
interdisciplinary Italian research centers. The data correspond to 50 patients classified in 5 groups of 10 subjects each: normal, hypertensive, post-MI, with congestive heart failure, and heart transplanted.

For each subject there are 24-hour Holter recordings, whose sample frequencies range from 128 to $1024 \mathrm{~Hz}$, according to the type of pathology.

\section{Results}

\subsection{Long-term analysis}

The analysis performed with the Diffusion Entropy technique yields very accurate fits as shown in Figs. 11 and 2. The average results for each group of patients is shown in table 1 .

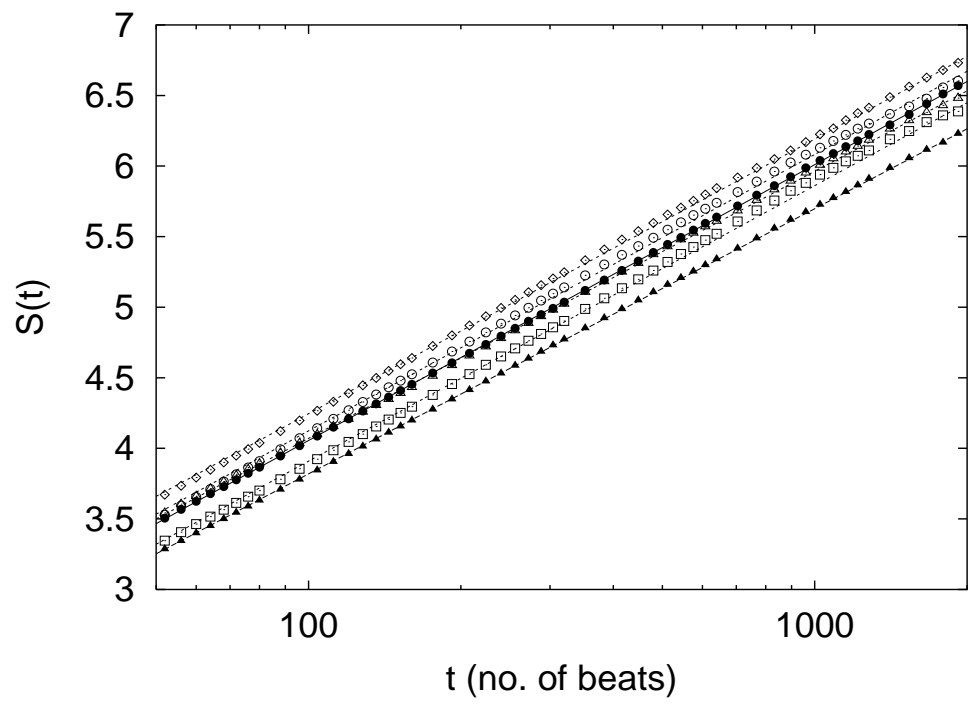

Fig. 1. Diffusion Entropy $\mathrm{S}(\mathrm{t})$ for healthy patients. The straights lines in loglinear scale represent best fits for Eq. (5)

As shown in table 1, the values of $\delta$ indicate very strong correlations for all the different groups. The CHF individuals, however, as previously shown in Ref. [2,6], are characterized by a somewhat smaller value. One of the main results of this paper is that, remarkably, the results from table 1 are independent of the coarse-graining parameter $s$. As we shall show later, this property can be accounted for by a simple intermittent model.

On the other hand, the coarse-graining parameter $s$ becomes very important in evaluating the correlation function $\Phi_{\tau}(j)$. In fact, different values of $s$ yield different series for $\tau$, while a large/small coarse graining leads to higher/lower 


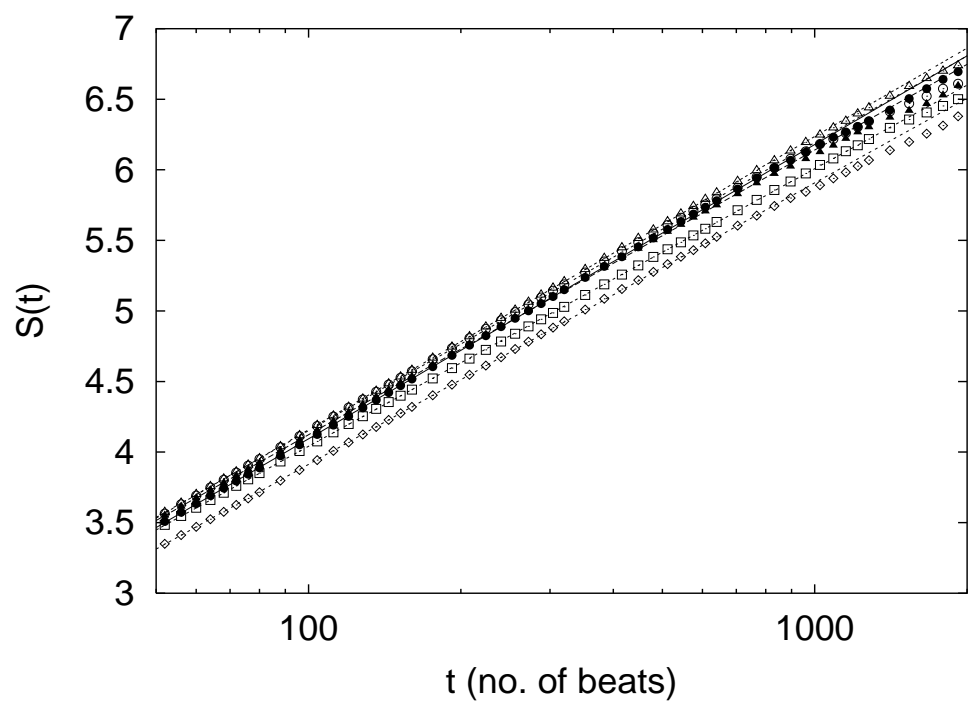

Fig. 2. Diffusion Entropy S(t) for patients hypertensive. The straights lines represent best fits for Eq. (5)

Table 1. Average value of $\delta$ for each group of patients

\begin{tabular}{|c|c|}
\hline Group & $\langle\delta\rangle$ \\
\hline healthy & $0.83 \pm 0.02$ \\
CHF & $0.80 \pm 0.05$ \\
post-MI & $0.85 \pm 0.04$ \\
transplanted & $0.86 \pm 0.08$ \\
hypertensive & $0.87 \pm 0.02$ \\
\hline
\end{tabular}


value for $\langle\tau\rangle$. We investigate the different correlation functions stemming from different coarse-graining values $s$. As earlier stated, in most cases the correlation function presents an abrupt drop going from $j=0$ to $j=1$. After this fast drop, it decays to zero very slowly, like an inverse-power law. In fact, the data are not clean enough to establish the inverse power law index with a good accuracy. This means that the DE is a much more efficient method to detect memory and much less ambiguous than the correlation function, and we can actually describe the meaning of $\delta$ making use of a general class of dynamical models. The correlation function $\Phi_{\tau}(j)$, on the other hand, depends on the details of the model and does not afford an easy way to derive it.

The numerical form of the correlation function $\Phi_{\tau}(j)$ is

$$
\Phi_{\tau}(j)=\left(1-\epsilon^{2}\right) \delta_{j, 0}+\epsilon^{2} C(j) .
$$

Here $\delta_{j, 0}$ denotes the Kronecker $\delta$ step, while the function $C(j)$ is a smooth function with the property $C(0)=1$. We account for the long-term properties, namely second term in the r. h. s of (9), as follows. We hypothesize that the heartbeat rate, on a rather long time scale, undergoes trends of acceleration and deceleration of slopes $\alpha$, whose duration length $\tau$ are asymptotically distributed with an inverse power law, with index $\mu^{\prime}$. We suppose that the consecutive trends are not correlated and consequenlty the scaling parameter $\delta$ is connected to $\mu^{\prime}$ via 海

$$
\delta=\frac{1}{\mu^{\prime}-1} .
$$

Here we sketch a proof of Eq. (10). First of all we define the distribution probability for the slope $\alpha, f(\alpha)$. We make the simplifying and plausible hypothesis that this distribution has no infinite moments. This restriction does not apply to the $\tau$-distribution, which has, on the contrary slow tails and diverging moments. Without loss of generality we assume the following form

$$
\psi(\tau)=\frac{A^{\mu^{\prime}-1}}{(A+\tau)^{\mu^{\prime}}},
$$

where $A=\langle\tau\rangle /\left(\mu^{\prime}-2\right)$. During one single trend of acceleration or deceleration the position of the walker $x_{l}(t)$ is displaced by a quantity

$$
z=\frac{|\alpha|}{s} \tau,
$$

which is the number of times the walker crosses the "box boundaries" defined by the coarse-graining procedure on $T_{i}$. Consequently, we can write the probability of a displacement $z$ that reads

$$
p(z)=\int_{0}^{\infty} \mathrm{d} \tau \int_{-\infty}^{\infty} \mathrm{d} \alpha \delta\left(z-\frac{\alpha \tau}{s}\right) \psi(\tau) f(\alpha) .
$$

After many trends it is straightforward to see, e. g. along the lines of Ref. [8], that the movement of our walker is essentially equivalent to a process where a 
walker, at regular time distances of duration $\langle\tau\rangle$, walks by a quantity $\Delta x \sim z$, distributed as

$$
\Pi(\Delta x)=\frac{s}{|\alpha|\langle\tau\rangle} \psi\left(\frac{s \Delta x}{|\alpha|}\right) .
$$

Since the function $\psi$ has a long tail, this latter process, according to the Generalized Central Limit Theorem [9], results in a Lévy Flight, which is a stable process with diverging central moments. The difficulty of dealing directly with the distribution (14) is due to the fact that $\alpha$ is itself a stochastic variable. However we can detect the asymptotic scaling property of the walker $x_{l}(t)$ by studying the first diverging moment of the distribution $p(z)$. To find this value we evaluate

$$
\left\langle z^{\gamma}\right\rangle=\int_{0}^{\infty} p(z) z^{\gamma} \mathrm{d} z
$$

The smallest value of $\gamma$ for which this integral diverges, called $\hat{\gamma}$, defines the scaling $\delta$ via $\delta=1 / \hat{\gamma}[9]$. Plugging Eq. (13) into (15), we obtain

$$
\left\langle z^{\gamma}\right\rangle=\int_{0}^{\infty} \mathrm{d} z \int_{0}^{\infty} \mathrm{d} \tau \int_{-\infty}^{\infty} \mathrm{d} \alpha \delta\left(z-\frac{\alpha \tau}{s}\right) \psi(\tau) f(\alpha) z^{\gamma} .
$$

Integrating over $z$, this result can be written as

$$
\left\langle z^{\gamma}\right\rangle=\left(\int_{0}^{\infty} \psi(\tau) \tau^{\gamma} \mathrm{d} \tau\right) \cdot F_{s},
$$

where

$$
F_{s}=\int_{-\infty}^{\infty} \mathrm{d} \alpha\left(\frac{|\alpha|}{s}\right)^{\gamma} f(\alpha) .
$$

This means that only the first factor might make $\left\langle\tau^{\gamma}\right\rangle$ divergent, thereby implying that $\hat{\gamma}=\mu^{\prime}-1$ and proving Eq. (10). This also proves that $\delta$ does not depend either on the coarse-graining parameter $s$ or on the distribution of $\alpha$, which in fact contribute only to $F_{s}$.

We now establish an interesting connection with the two-walkers model of Ref. [6 as follows. We assume that the trajectory $T_{i}$ is a zig-zag path. Each straight line of this path is determined by the slope $\alpha_{j}$, which connects the persistency time $\tau_{j}$ to the coarse-grain parameter $s$, by

$$
\alpha_{j}=\frac{s}{\tau_{j}} .
$$

If the straight line portion of the zig-zag curve has length $l_{j}$, we have about $l_{j} \alpha_{j} / s$ identical values of $\tau_{j}$. These are the pseudoevents of the two-walkers model of Ref. [6]. 


\subsection{Short-term analysis}

Of course this crude model can only describe the long-time regulation of the heartbeat dynamics and has to be supplemented with middle- and short-time corrections so as to take into account the abrupt fall corresponding to the first term in the r.h.s. of Eq. (9). Indeed short-term control mechanisms are due to the activity of the autonomic nervous system which presents a very short time scale.

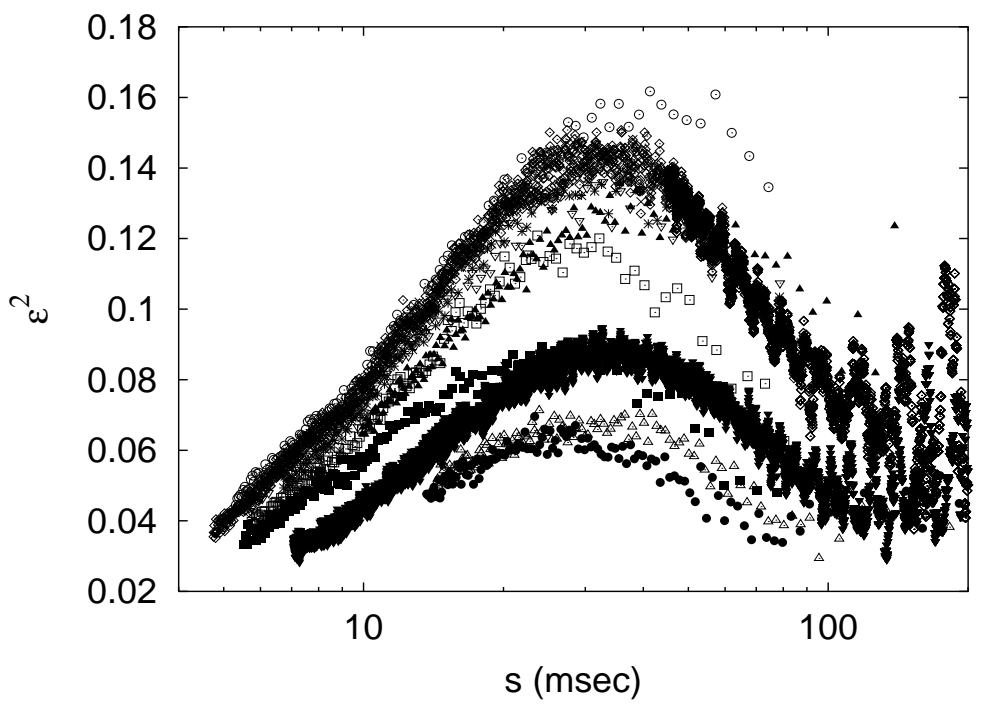

Fig. 3. Short-time correlation parameter $\epsilon^{2}$ as a function of the coarse-graining resolution $s$ for all the the healthy subjects. Two of them are plotted with a much higher number of points, to show both the subtle erratic dependence and the "continuous" one.

We use a short-time (local) perturbation model which does not destroy longtime properties, in a way that is similar to the point-like mutations affecting the long-range correlations in DNA sequences [10]. The value of $\epsilon^{2}$ does in fact depend on the value of $s$ and therefore we compute $\epsilon^{2}(s)$ in order to investigate the properties of these control mechanisms. Surprisingly enough, the function $\epsilon^{2}(s)$ show a quite universal feature for all healthy and hypertensive individuals: we observe a steep increase as $s$ goes from few msec up to $s_{\max } \cong 30 \mathrm{msec}$ where we have a maximum. After that we have a slow decrease until $200 \mathrm{msec}$. The most striking result is that, even if the maximum value is quite different among the various individuals, the values of $s_{\max }$ seems to be universal, the variability among different patients being lower than 5 msec. This result is shown in Fig.3. This feature is somewhat visible (always for $s_{\max }=30 \mathrm{msec}$ ) also in post-

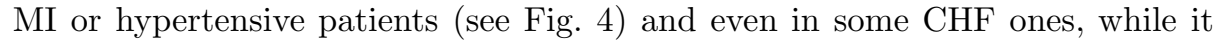


is completely absent in some CHF patients and in all the heart transplanted. Results for these latter groups are shown in Fig. 5 .

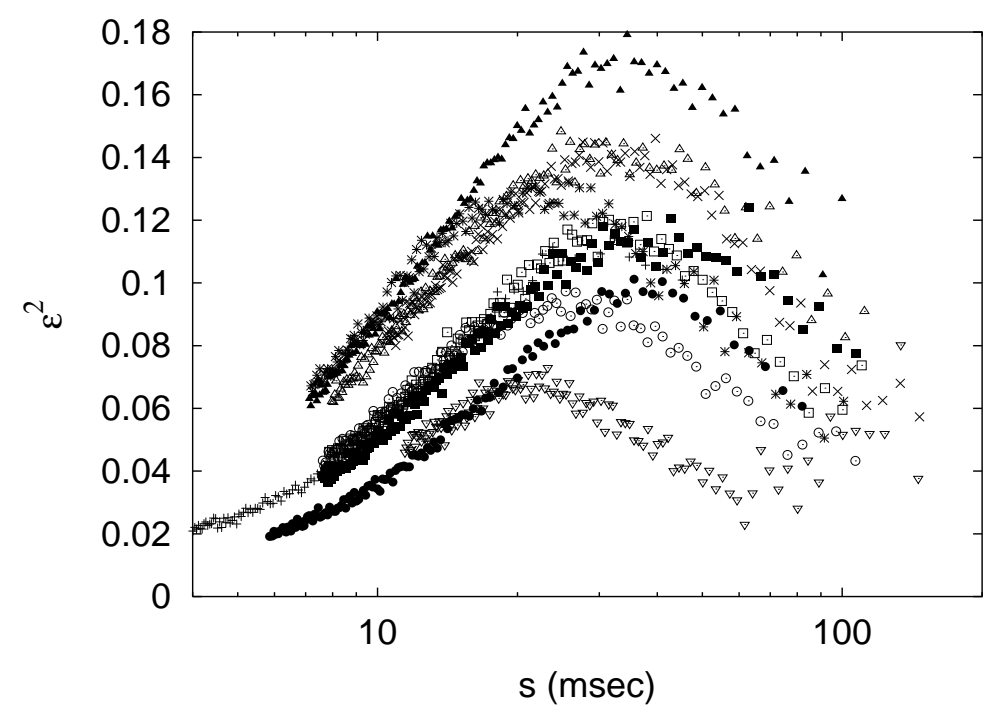

Fig. 4. Short-time correlation parameter $\epsilon^{2}$ as a function of the coarse-graining resolution $s$ for 5 hypertensive patients and 5 post-MI patients. We plot only half of these groups for plot clarity.

To the best of our knowledge, the interpretation of $s_{\max }$ is not easily explained in physiological terms. With the due caution, we may intepret this scale as an intrinsic resolution for the assessment of the heartbeat rate from the control system. In other words there should be a natural coarse graining in the physiological determination of the interbeat time distance. The fact that this behavior is missing in the case of heart transplanted patients seems to imply the action of the autonomic system. Anyway, the measured timescales rule out the possibility that this maximum is due to the sampling rate of the measuring apparatus. We conclude that more work has to be done to explain this effect throughfully. Finally, from a methodological point of view we use this property to evaluate in an objective way a value for the parameter $\epsilon^{2}$, which is a measure of the regularity of the interbeat time accelerations $\alpha$ during the trends of variation.

At this point, as done in Ref. [6], we can plot the value of $\delta$, independent of our coarse graining, as a function of our objectively defined value of $\epsilon^{2}$. The result is shown in Fig. 6. We recall that both parameters reflect memory, the former being associated with long-range fractal properties, and the latter with short-time persistency in the heart acceleration patterns. This latter behavior 


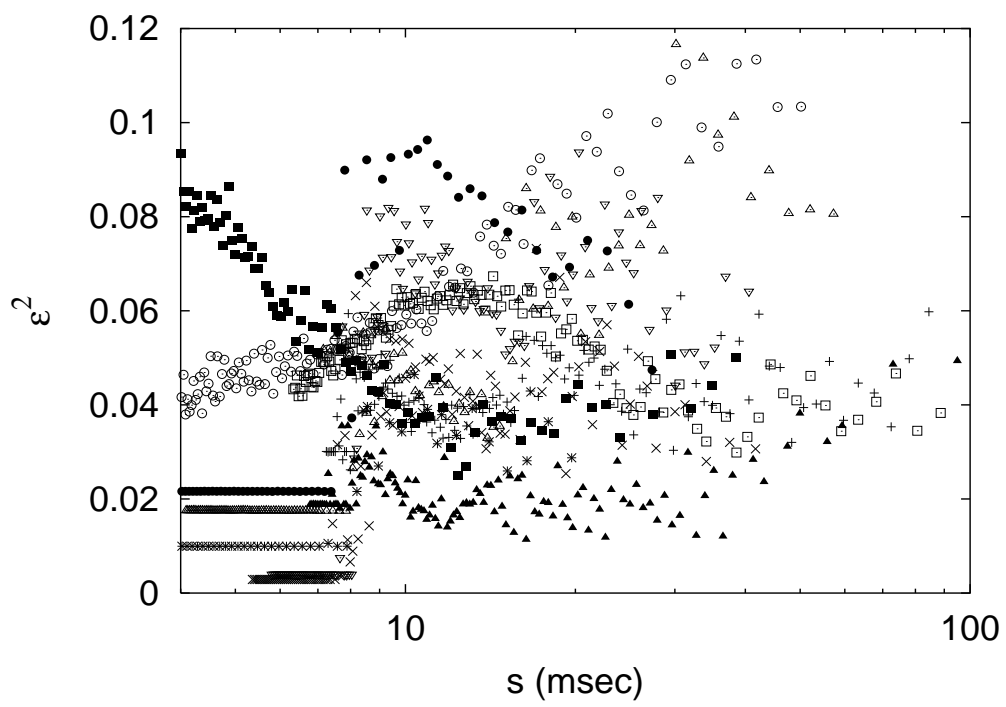

Fig. 5. Short-time correlation parameter $\epsilon^{2}$ as a function of the coarse-graining resolution $s$ for all 10 transplanted patients. The structure of the previous figures is absent.

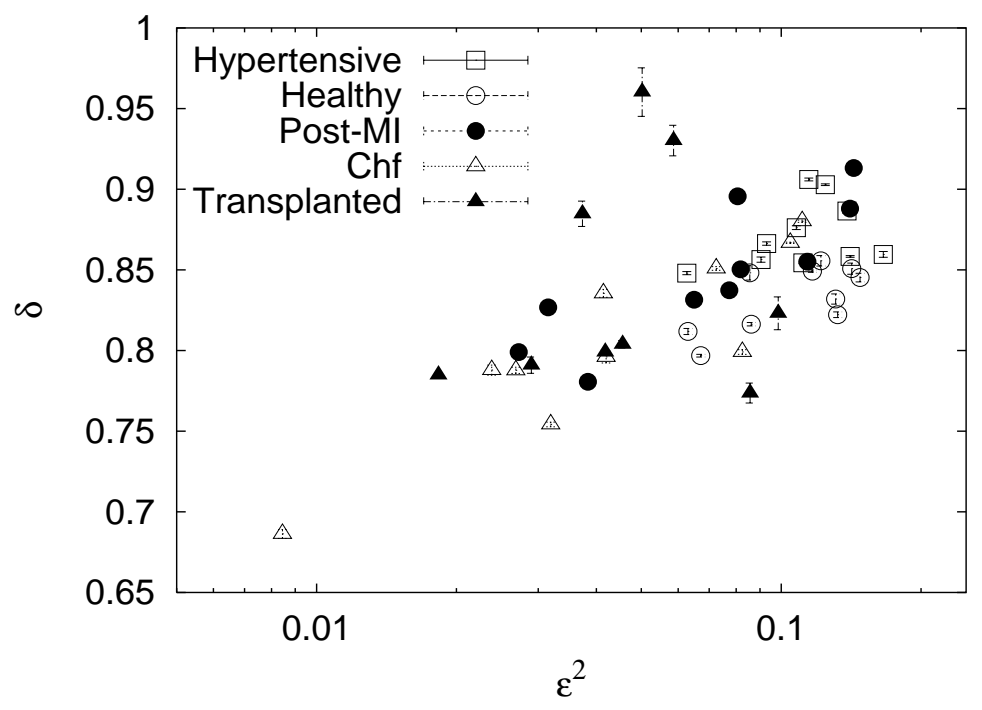

Fig. 6. Bidimensional plot of $\delta\left(\epsilon^{2}\right)$ for all the 50 subjects.

is certainly connected with multifractal properties, as reported e.g. in Ref. [11]. Arguably, the proposed treatment here proposed is more intuitive. 
Fig. 6 shows the $\left\{\epsilon^{2}, \delta\right\}$ scatter plot for all 50 subjects. Even though there is no satisfactory statistical discrimination among groups, maybe due to a variety of factors like the limited number of patients, differences in illness seriousness and differences in therapy, we observe that healthy individuals are concentrated in a small portion of the graph, in agreement with the results of Ref. [6]. Hypertensive patients, too, have their peculiar region, characterized by an higher value of $\delta$. Other pathologies seem to occupy broader regions. A significant portion of pathological individuals occupy the "healthy zone", and these may be associated to less serious conditions. Actually, preliminary results (unpublished) on an enlarged group of CHF patients seem to suggest a correlation between serious condition and the absence/distorsion of the $\epsilon^{2}(s)$ peak at $30 \mathrm{msec}$. These patients are also characterized by a smaller value of $\epsilon^{2}(s)$ and they are therefore mostly outside the "healthy" zone.

\section{Conclusions}

In conclusion, this study allows us to propose, on the basis of a simple statistical analysis, a series of conjectures to be accurately tested by further studies. The physiological long-range memory, probably due to a cascade of mechanisms at different timescales, can be characterized by two parameters, the scaling $\delta$ of the underlying infinite-memory process, and the weight $\epsilon^{2}$, which gives information of the relative importance of this latter process versus short-time mechanisms. The presence of the 30 milliseconds peak of Fig. 3, which allows an objective definition of $\epsilon^{2}$ seems to be physiological and corresponds to the presence of a fast control mechanism, which has to react in a time shorter than few heartbeats to properly explain the abrupt fall of the correlation functions. This control mechanism seems to act with a special time resolution: a "grid" of about 30 milliseconds. The absence, distorsion, or shift of this internal resolution may reflect a pathological condition and is often associated with a lower degree of long-time memory.

Finally, we stress that the coarse-graining procedure adopted herein yields results that are independent of the coarse-graining parameter, making this analysis robust. We believe that this analysis can be applied with success to a variety of time series with both long- and short-time structures, and even to nonstationary signals. Results stemming form our procedure may thereby provide additional information useful in many diagnostic and prognostic problems. In general, we envisage our procedure as an automatic classifier for those complex sequences that are not easily dealt with, using usual statistical learning methods. Applications may range from natural-language semantic classifiers based on discourse dynamics to predictors of catastrophic events [5].

Aknowledgements. We gratefully acknowledge financial support from the Army Research Office through Grant DAAD 19-02-0037. One of the authors (P. A.) acknowledges European Commission POESIA project (IAP2117/27572) for financial support. L. P. acknowledges ENEL Research Grant 3000021047 for support. 


\section{References}

1. Shlesinger, M.F.: Fractal time and 1/f noise in complex system. Ann. NY Acad. Sci. 504 (1987) 214-228; Bassingthwaighte, J.B., Liebovitch, L.S., West, B.J.: Fractal Physiology. Oxford University Press, New York (1994)

2. Ashkenazy, Y., Ivanov, P.Ch., Havlin, S., Peng, C.-K., Goldberger, A.L., Stanley, H.E.: Magnitude and Sign Correlation in Heartbeat Fluctuations. Phys. Rev. Lett. 86 (2001) 1900-1903

3. Balocchi, R., Barbi, M., Carpeggiani, C., Chillemi, S., Di Garbo, A., Michelassi, C.: Complexity and Predictability of the Heartbeat Time Series in Transplanted Subjects. Int. J. Bifurcation and Chaos 7 (1997) 759-767

4. Grigolini, P., Palatella, L., Raffaelli, G.: Asymmetric Anomalous Diffusion: an Efficient Way to Detect Memory in Time Series. Fractals 9 (2001) 439-449

5. Allegrini, P. ,Grigolini, P., Hamilton, P., Palatella, L., Raffaelli, G., Virgilio, M.: Facing nonstationarity condition with a new indicator of Entropy Increase: the Cassandra Algorithm. In: Novak, M.N. (ed.): Emergent Nature. World Scientific, Singapore (2002) 173-184

6. Allegrini, P., Grigolini, P., Hamilton, P., Palatella L., Raffaelli, G.: Memory Beyond Memory in Heart Beating, a Sign of a Healthy Physiological Condition. Phys. Rev. E 65 (2002) 041926-041930

7. Signorini, M.G., Sassi, R., Cerutti, S.: Working on the Noltisalis Database: measurement of nonlinear properties in heart rate variability signals. In: Proc. of the 23th Annual Conf. of the IEEE-EMBS, Istanbul, Turkey, Oct 24-28 (2001)

8. Annunziato M., Grigolini, P.: Stochastic versus Dynamic Approach to Lévy Statistics in the Presence of an External Perturbation. Phys. Lett. A 269 (2000) 31-39

9. B. V. Gnedenko, B.V., Kolmogorov, A.N.: Limit Distributions for Sums of Independent Random Variables. Addison Wesley, Cambridge MS (1954)

10. Allegrini, P., Buiatti, M., Grigolini, P., West, B.J.: Fractional Brownian Motion as a Nonstationary Process: an Alternative Paradigm for DNA sequences. Phys. Rev. E 57 (1998) 4558-4567

11. Ivanov, P.Ch., Nunes Amaral, L.A., Goldberger, A.L., Havlin, S. Rosenblum, M.G., Struzik, Z.R., Stanley, H.E.: Multifractality in Human Heartbeat Dynamics. Nature 399 (1999) 461-463 